TRANSACTIONS OF THE

AMERICAN MATHEMATICAL SOCIETY

Volume 355, Number 2, Pages 637-653

S 0002-9947(02)03110-0

Article electronically published on September 19, 2002

\title{
ON PARTITIONING THE ORBITALS OF A TRANSITIVE PERMUTATION GROUP
}

\author{
CAI HENG LI AND CHERYL E. PRAEGER
}

\begin{abstract}
Let $G$ be a permutation group on a set $\Omega$ with a transitive normal subgroup $M$. Then $G$ acts on the $\operatorname{set} \operatorname{Orbl}(M, \Omega)$ of nontrivial $M$-orbitals in the natural way, and here we are interested in the case where $\operatorname{Orbl}(M, \Omega)$ has a partition $\mathcal{P}$ such that $G$ acts transitively on $\mathcal{P}$. The problem of characterising such tuples $(M, G, \Omega, \mathcal{P})$, called TODs, arises naturally in permutation group theory, and also occurs in number theory and combinatorics. The case where $|\mathcal{P}|$ is a prime-power is important in algebraic number theory in the study of arithmetically exceptional rational polynomials. The case where $|\mathcal{P}|=2$ exactly corresponds to self-complementary vertex-transitive graphs, while the general case corresponds to a type of isomorphic factorisation of complete graphs, called a homogeneous factorisation. Characterising homogeneous factorisations is an important problem in graph theory with applications to Ramsey theory. This paper develops a framework for the study of TODs, establishes some numerical relations between the parameters involved in TODs, gives some reduction results with respect to the $G$-actions on $\Omega$ and on $\mathcal{P}$, and gives some construction methods for TODs.
\end{abstract}

\section{INTRODUCTION}

Each transitive permutation group $M$ on a set $\Omega$ has a natural induced action on the set

$$
\Omega^{(2)}=(\Omega \times \Omega) \backslash\{(\alpha, \alpha) \mid \alpha \in \Omega\}=\{(\alpha, \beta) \mid \alpha \neq \beta \in \Omega\},
$$

given by $(\alpha, \beta)^{x}=\left(\alpha^{x}, \beta^{x}\right)$ for $\alpha, \beta \in \Omega$ with $\alpha \neq \beta$ and $x \in M$. The $M$-orbits in $\Omega^{(2)}$ are called $M$-orbitals on $\Omega$, and a partition $\mathcal{P}$ of $\Omega^{(2)}$ is called an $M$ orbital decomposition if each class $P \in \mathcal{P}$ is a union of one or more $M$-orbitals. Let $\operatorname{Orbl}(M, \Omega)$ denote the set of $M$-orbits in $\Omega^{(2)}$. In this paper we investigate the situation where $M<G \leq \operatorname{Sym}(\Omega)$, and $G$ induces a transitive action on an $M$-orbital decomposition $\mathcal{P}$ of $\Omega^{(2)}$; that is to say, we assume

(i) for all $P \in \mathcal{P}$ and $g \in G, P^{g} \in \mathcal{P}$,

(ii) for $P, P^{\prime} \in \mathcal{P}$, there exists $g \in G$ such that $P^{g}=P^{\prime}$, and

(iii) $\mathcal{P}$ is refined by $\operatorname{Orbl}(M, \Omega)$.

If these conditions hold, then we call the tuple $(M, G, \Omega, \mathcal{P})$ a transitive orbital decomposition, or a $T O D$ for short. The cardinalities $|\Omega|$ and $|\mathcal{P}|$ are called the degree and the index of the TOD, and sometimes we refer to $(M, G, \Omega, \mathcal{P})$ as a $k$-TOD if $k=|\mathcal{P}|$.

Received by the editors October 23, 2001.

2000 Mathematics Subject Classification. Primary 20B15, 20B30, 05C25.

This work forms a part of an Australian Research Council grant project.

(C)2002 American Mathematical Society 
Interest in transitive orbital decompositions has arisen from several different areas. The case $k=2$ corresponds to self-complementary graphs, which are discussed in more detail below. The case where $k$ is a prime-power arose also in algebraic number theory in the study of arithmetically exceptional rational polynomials [11]. (See 7] for the more general context of these investigations.)

Assume that $k=2$. Then the two classes $P, P^{\prime}$ in $\mathcal{P}$ can be regarded as the edge sets of directed graphs (digraphs) $\Gamma=(\Omega, P)$ and $\Gamma^{\prime}=\left(\Omega, P^{\prime}\right)$ such that the group $M$ is a vertex-transitive automorphism group of both $\Gamma$ and $\Gamma^{\prime}$, and each $g \in G$ that interchanges $P$ and $P^{\prime}$ induces a graph isomorphism between $\Gamma$ and $\Gamma^{\prime}$. Thus $\Gamma, \Gamma^{\prime}$ is a pair of self-complementary vertex-transitive digraphs. Moreover, if $\mathcal{P}$ is symmetric, in the sense that each $P \in \mathcal{P}$ is equal to its reverse $P^{*}=\{(\beta, \alpha) \mid(\alpha, \beta) \in P\}$, and if $\mathcal{P}$ has index $k=2$, then the two graphs $\Gamma, \Gamma^{\prime}$ may be considered as undirected self-complementary graphs, namely, $\Gamma=(\Omega, E)$ and $\Gamma^{\prime}=\left(\Omega, E^{\prime}\right)$, where the edge sets $E, E^{\prime}$ are the sets of unordered pairs $\{\alpha, \beta\}$ from $\Omega$ with $(\alpha, \beta)$ in $P, P^{\prime}$, respectively.

The study of vertex-transitive self-complementary graphs began with a construction of a family of self-complementary circulant graphs given by H. Sachs [24]. Vertex-transitive self-complementary graphs have received considerable attention in the literature, see for example [14, 17, 18, 20, 25, 27, and they have been used to investigate Ramsey numbers [3, 4, 5. Most of the known vertex-transitive selfcomplementary graphs are Cayley graphs, see for example [14, 18, 25, 19, 23, the first infinite family of vertex-transitive self-complementary graphs that are not Cayley graphs was constructed recently in [17]. With regard to TODs of arbitrary index $k$, we give necessary and sufficient conditions for the existence of a $k$-TOD in Proposition [3.3, and the proof of this result includes a general construction for them. Explicit constructions are given in Section [6]

A lot of effort was expended in determining the positive integers $n$ such that there exist vertex-transitive self-complementary graphs with $n$ vertices, see [1, 8, 15, 20. 27, and recently, Muzychuk [20] completely determined such positive integers. One of the major results of this paper, Theorem 1.1, is a generalisation of Muzychuk's result. A $k$-TOD $(M, G, \Omega, \mathcal{P})$ is called cyclic if the transitive permutation group $G^{\mathcal{P}}$ induced by $G$ on $\mathcal{P}$ is cyclic. Thus a pair of vertex-transitive self-complementary digraphs corresponds to a cyclic 2-TOD. Our result classifies the possibilities for the degree of a cyclic $k$-TOD, and the case $k=2$ is the result of Muzychuk 20].

Theorem 1.1. Let $k$ be an integer such that $k>1$, and let $n=r_{1}^{d_{1}} r_{2}^{d_{2}} \ldots r_{m}^{d_{m}}$, where the $r_{i}$ are distinct primes, $d_{i} \geq 1$, and $m \geq 1$. Then

(i) there exists a cyclic $k$-TOD of degree $n$ if and only if, for all $i=1, \ldots, m$,

$$
r_{i}^{d_{i}} \equiv 1(\bmod k) \text {; }
$$

(ii) there exists a cyclic symmetric $k$-TOD of degree $n$ if and only if, for all $i=1,2, \ldots, m$,

$$
\begin{aligned}
& r_{i}^{d_{i}} \equiv 1(\bmod 2 k) \text { if } r_{i} \text { is odd, or } \\
& r_{i}^{d_{i}} \equiv 1(\bmod k) \text { if } r_{i}=2 .
\end{aligned}
$$

For a transitive group $M \leq \operatorname{Sym}(\Omega)$, let $M^{(2)}$ denote the 2-closure of $M$ in the sense of Wielandt; that is, $M^{(2)}$ is the largest subgroup of $\operatorname{Sym}(\Omega)$ with the same orbits as $M$ in $\Omega^{(2)}$. One way that a subgroup $G \leq \operatorname{Sym}(\Omega)$ may leave invariant a partition refined by $\operatorname{Orbl}(M, \Omega)$ is if $G$ permutes the $M$-orbitals setwise. We show 
(Proposition 2.1) that a subgroup $G \leq \operatorname{Sym}(\Omega)$ leaves the set $\operatorname{Orbl}(M, \Omega)$ invariant (that is, $\Delta^{g} \in \operatorname{Orbl}(M, \Omega)$ for all $\Delta \in \operatorname{Orbl}(M, \Omega)$ and all $\left.g \in G\right)$ if and only if $G$ normalises $M^{(2)}$. However, whether or not $G$ leaves $\operatorname{Orbl}(M, \Omega)$ invariant in a TOD $(M, G, \Omega, \mathcal{P})$, we may always replace $M$ by the kernel $\hat{M}$ of the action of $G$ on $\mathcal{P}$, and thereby obtain a TOD $(\hat{M}, G, \Omega, \mathcal{P})$ with $\hat{M}$ normal in $G$. From Subsection 2.2 onwards we shall always assume that $M$ is normalised by $G$. We begin by presenting in Section 2 several elementary properties of TODs and in Section 3 constructions of new TODs from a given TOD. Some of these constructions yield examples of cyclic TODs.

If $(M, G, \Omega, \mathcal{P})$ is a $k$-TOD and $\mathcal{B}$ is a nontrivial block system for $G$ in $\Omega$, then the induced structure $\left(M_{B}^{B}, G_{B}^{B}, B, \mathcal{P}_{B}\right)$ on a block $B \in \mathcal{B}$ is also a $k$-TOD (Lemma 4.1), but there seems to be no natural induced TOD corresponding to the actions of $M$ and $G$ on $\mathcal{B}$ (see Subsection 4.2). However, for cyclic $k$-TODs, we are able to induce a cyclic $k$-TOD for the quotient action on $\mathcal{B}$.

Theorem 1.2. Let $(M, G, \Omega, \mathcal{P})$ be a cyclic $k$-TOD. Then for any $G$-invariant partition $\mathcal{B}$ of $\Omega$, there exists a partition $\mathcal{Q}$ of $\mathcal{B}^{(2)}$ such that $\left(M^{\mathcal{B}}, G^{\mathcal{B}}, \mathcal{B}, \mathcal{Q}\right)$ is a cyclic $k-T O D$.

This theorem will be proved in Section 3. It raises the question of classifying cyclic $k$-TODs $(M, G, \Omega, \mathcal{P})$ with $G$ primitive on $\Omega$. For the case where $k$ is a primepower, a classification is given in [10]. In Section 4 we give several constructions of cyclic TODs proving the existence assertions of Theorem 1.1. and we complete the proof of Theorem 1.1 in Section 5.

1.1. Further graph-theoretic links. From another viewpoint, a symmetric TOD of degree $n$ is a special type of isomorphic factorisation of the complete graph $K_{n}$ on $n$ vertices. An isomorphic factorisation of $K_{n}$ with vertex set $V$ is a decomposition $\left\{E_{1}, \ldots, E_{k}\right\}$ of the unordered pairs of vertices such that the $k$ graphs $\left(V, E_{1}\right), \ldots,\left(V, E_{k}\right)$ are pairwise isomorphic; the graphs $\left(V, E_{i}\right)$ are called the factors of the factorisation. Isomorphic factorisations of complete graphs have been investigated for a long time, see for instance [12, 13. If $(M, G, \Omega, \mathcal{P})$ is a symmetric $k$-TOD of degree $n$ and if $E_{i}=\left\{\{x, y\} \mid(x, y) \in P_{i}\right\}$ where $\mathcal{P}=\left\{P_{1}, \ldots, P_{k}\right\}$, then $\left\{E_{1}, \ldots, E_{n}\right\}$ is an isomorphic factorisation of $K_{n}$ with vertex set $\Omega$ with the additional property that the group $G$ permutes $\left\{E_{1}, \ldots, E_{k}\right\}$ transitively. Thus, isomorphisms between each pair $\left(V, E_{i}\right)$ and $\left(V, E_{j}\right)$ can be induced by elements of $G$. Isomorphic factorisations of $K_{n}$ with this property are called homogeneous factorisations.

We end this section with a discussion of two special classes of TODs. Let $(M, G, \Omega, \mathcal{P})$ be a $k$-TOD such that $\mathcal{P}=\operatorname{Orbl}(M, \Omega)$, that is, $\mathcal{P}$ is a trivial partition of $\operatorname{Orbl}(M, \Omega)$. It then follows that $G$ is a 2-transitive permutation group on $\Omega$, and $M$ is a permutation group of rank $k+1$. By Proposition 2.1, we may assume that $G$ contains $M^{(2)}$, and then $M^{(2)}$ is a normal subgroup of $G$ of rank $k+1$. Inspecting the classification of 2-transitive permutation groups, see [2], we see that either $G$ is affine, or $M \cong \mathrm{PSL}_{2}(8)$ and $G \cong \operatorname{Aut}\left(\mathrm{PSL}_{2}(8)\right)$. The latter indeed gives rise to a 3 -TOD $(M, G, \Omega, \mathcal{P})$ such that $\mathcal{P}=\operatorname{Orbl}(M, \Omega)$, which is symmetric and has degree 28. This, in particular, shows that the complete graph $K_{28}$ may be factorised into three isomorphic arc-transitive graphs of valency 9. Further, this, together with Theorem 1.1. also shows that a complete graph $K_{n}$ having a nontrivial homogeneous factorisation with $M$-arc-transitive factors implies that $n$ is a 
prime-power or $n=28$. The special case where $k=2$ corresponds to arc-transitive self-complementary graphs, which are classified in [21, 28].

A more general interesting class of TODs is the class of homogeneous factorisations of complete graphs with edge-transitive factors. This corresponds to the class of symmetric $k$-TODs $(M, G, \Omega, \mathcal{P})$ such that each $P_{i} \in \mathcal{P}$ is an orbital, or a union of two paired orbitals of $M^{\Omega}$. In this case, it follows that we can take $G$ to be a 2-homogeneous permutation group on $\Omega$ with a transitive normal subgroup $M^{(2)}$ of rank $k+1$. The TOD arising from $\mathrm{PSL}_{2}(8)$ is also an example for this case.

In subsequent work [16], a complete description will be given of homogeneous factorisations of complete graphs with arc-transitive or edge-transitive factors.

\section{General properties of TODs}

2.1. On the normality of $M$. Some TODs $(M, G, \Omega, \mathcal{P})$ arise with the group $G$ having an induced action on the $\operatorname{set} \operatorname{Orbl}(M, \Omega)$ of $M$-orbitals. This condition is not part of the definition of a TOD. However, we do not have any examples where it does not hold. We first prove the result mentioned in the introduction, which relates this property to the 2-closure of $M$.

Proposition 2.1. Let $M$ be a transitive permutation group on a set $\Omega$. Then a subgroup $G \leq \operatorname{Sym}(\Omega)$ leaves $\operatorname{Orbl}(M, \Omega)$ invariant if and only if $G$ normalises $M^{(2)}$.

Proof. Suppose that $G$ normalises $M^{(2)}$. Let $g \in G$ and $\Delta \in \operatorname{Orbl}(M, \Omega)$. We need to prove that $\Delta^{g} \in \operatorname{Orbl}(M, \Omega)$. For $x \in M$, we have $x^{g^{-1}} \in M^{(2)}$, and hence $\Delta^{g x g^{-1}}=\Delta^{x^{g^{-1}}}=\Delta$. So $\Delta^{g x}=\Delta^{g}$, that is, $\Delta^{g}$ is $M$-invariant. For any $(\alpha, \beta),(\gamma, \delta) \in \Delta$, there exists $x \in M$ such that $(\alpha, \beta)^{x}=(\gamma, \delta)$. Now $g^{-1} x g \in$ $M^{(2)},\left(\alpha^{g}, \beta^{g}\right),\left(\gamma^{g}, \delta^{g}\right) \in \Delta^{g}$, and $\left(\alpha^{g}, \beta^{g}\right)^{g^{-1} x g}=\left(\gamma^{g}, \delta^{g}\right)$. It follows that $M^{(2)}$ is transitive on $\Delta^{g}$, and so $\Delta^{g}$ is an $M^{(2)}$-orbital. Thus by the definition of $M^{(2)}$, $\Delta^{g} \in \operatorname{Orbl}(M, \Omega)$, and hence $G$ leaves $\operatorname{Orbl}(M, \Omega)$ invariant.

Conversely, suppose that $G$ leaves $\operatorname{Orbl}(M, \Omega)$ invariant. Let $x \in M^{(2)}$ and $g \in G$. We claim that $x^{g} \in M^{(2)}$. Take an arbitrary element $\Delta \in \operatorname{Orbl}(M, \Omega)$. Then $\Delta^{g^{-1}} \in \operatorname{Orbl}(M, \Omega)$, and hence $\left(\Delta^{g^{-1}}\right)^{x}=\Delta^{g^{-1}}$. Therefore, $\Delta^{x^{g}}=\Delta^{g^{-1} x g}=\Delta$, and so $x^{g}$ fixes every element of $\operatorname{Orbl}(M, \Omega)$. Thus $x^{g} \in M^{(2)}$, and so $G$ normalises $M^{(2)}$.

If $(M, G, \Omega, \mathcal{P})$ is a $k$-TOD and $G$ normalises $M^{(2)}$, then $\left(M^{(2)} \cap G, G, \Omega, \mathcal{P}\right)$ is also a $k$-TOD, and by Lemma 2.1, $M^{(2)} \cap G$ is a normal subgroup of $G$. Whether or not this is the case, if $\hat{M}$ is the kernel of the action of $G$ on $\mathcal{P}$, then $(\hat{M}, G, \Omega, \mathcal{P})$ is a $k$-TOD. We will assume from now on that, for a $k$-TOD $(M, G, \Omega, \mathcal{P})$, the group $M$ is a normal subgroup of $G$. Moreover, we note the following.

Lemma 2.2. Let $(M, G, \Omega, \mathcal{P})$ be a $k$-TOD, and let $K$ be the kernel of the $G$ action on $\mathcal{P}$. Assume that $N$ is a normal subgroup of $G$ such that $N \leq K$ and $N$ is transitive on $\Omega$. Then $(N, G, \Omega, \mathcal{P})$ is a $k$-TOD.

Proof. This is clear from the definition of TODs.

2.2. TODs and partitions of $\Omega$. Choose a point $\omega \in \Omega$. Now we give a relation between a partition of $\Omega^{(2)}$ and a partition of $\Omega \backslash\{\omega\}$. For a partition $\mathcal{P}=\left\{P_{1}, P_{2}, \ldots, P_{k}\right\}$ of $\Omega^{(2)}$, let $P_{i}(\omega)=\left\{\omega^{\prime} \in \Omega \mid\left(\omega, \omega^{\prime}\right) \in P_{i}\right\}$, and let 
$\mathcal{P}(\omega)=\left\{P_{1}(\omega), P_{2}(\omega), \ldots, P_{k}(\omega)\right\}$. Let $M$ be a transitive permutation group on $\Omega$, and let $\mathcal{P}=\left\{P_{1}, P_{2}, \ldots, P_{k}\right\}$ be a partition of $\Omega^{(2)}$ refined by $\operatorname{Orbl}(M, \Omega)$. Then $\mathcal{P}(\omega)$ is a partition of $\Omega \backslash\{\omega\}$ refined by the set of $M_{\omega}$-orbits in $\Omega \backslash\{\omega\}$. Conversely, if $\mathcal{P}(\omega)$ is a partition of $\Omega \backslash\{\omega\}$ refined by the set of $M_{\omega}$-orbits in $\Omega \backslash\{\omega\}$, then we obtain a partition $\mathcal{P}$ of $\Omega^{(2)}$ refined by $\operatorname{Orbl}(M, \Omega)$, by defining $\mathcal{P}=\left\{P_{1}, \ldots, P_{k}\right\}$ with $P_{i}=\left\{\left(\omega, \omega^{\prime}\right)^{g} \in \Omega^{(2)} \mid \omega^{\prime} \in P_{i}(\omega), g \in M\right\}$. The next lemma shows that for an overgroup $G$ of $M$ that leaves $\mathcal{P}$ invariant, the $G$-action on $\mathcal{P}$ is equivalent to the $G_{\omega}$-action on $\mathcal{P}(\omega)$. Whenever a group $G$ has an action on a set $\mathcal{P}$ we shall denote by $G^{\mathcal{P}}$ the permutation group on $\mathcal{P}$ induced by $G$.

Lemma 2.3. Let $M$ be a transitive permutation group on $\Omega$, and suppose that $\mathcal{P}$ is a partition of $\Omega^{(2)}$ refined by $\operatorname{Orbl}(M, \Omega)$. Let $G$ be such that $M \triangleleft G \leq \operatorname{Sym}(\Omega)$, and let $\omega \in \Omega$. Then

(1) $\mathcal{P}$ is $G$-invariant if and only if $\mathcal{P}(\omega)$ is $G_{\omega}$-invariant;

(2) in the case where $\mathcal{P}$ is $G$-invariant, $G^{\mathcal{P}}=G_{\omega}^{\mathcal{P}}$ and the $G_{\omega}$-actions on $\mathcal{P}$ and $\mathcal{P}(\omega)$ are equivalent; in particular, $G$ is transitive on $\mathcal{P}$ if and only if $G_{\omega}$ is transitive on $\mathcal{P}(\omega)$, and thus $(M, G, \Omega, \mathcal{P})$ is a TOD if and only if $G_{\omega}$ is transitive on $\mathcal{P}(\omega)$.

Proof. Since $M$ is transitive on $\Omega, G=M G_{\omega}$, and thus each element $x \in G$ may be written as $x=g y$ for some $g \in M$ and some $y \in G_{\omega}$. Then for $P_{i}, P_{j} \in \mathcal{P}$, $P_{i}^{x}=P_{i}^{y}=P_{j}$ if and only if $P_{i}(\omega)^{y}=P_{j}(\omega)$. Thus $\mathcal{P}$ is $G$-invariant if and only if $\mathcal{P}(\omega)$ is $G_{\omega}$-invariant. Moreover, since $G=M G_{\omega}$, we have $G^{\mathcal{P}}=G_{\omega}^{\mathcal{P}}$ and the $G_{\omega^{-}}$ action on $\mathcal{P}$ is equivalent to the $G_{\omega}$-action on $\mathcal{P}(\omega)$. In particular, $G$ is transitive on $\mathcal{P}$ if and only if $G_{\omega}$ is transitive on $\mathcal{P}(\omega)$.

2.3. A congruence involving $n$ and $k$. Lemma 2.3 implies a congruence relation that must be satisfied by the parameters $n$ and $k$ for $k$-TODs of degree $n$.

Lemma 2.4. Let $(M, G, \Omega, \mathcal{P})$ be a $k$-TOD, where $\mathcal{P}=\left\{P_{1}, P_{2}, \ldots, P_{k}\right\}$. Then for each $G_{\omega}$-orbit $\Delta$ in $\Omega \backslash\{\omega\},\left\{\Delta \cap P_{i}(\omega) \mid 1 \leq i \leq k\right\}$ is a $G_{\omega}$-invariant partition of $\Delta$; in particular, $k$ divides $|\Delta|$.

Proof. By definition, $\mathcal{P}$ is a $G$-invariant partition of $\Omega^{(2)}$. Thus by Lemma 2.3 $\mathcal{P}(\omega)$ is a $G_{\omega}$-invariant partition of $\Omega \backslash\{\omega\}$. It follows, since $G_{\omega}$ fixes $\Delta$ setwise, that $\left\{\Delta \cap P_{i}(\omega) \mid 1 \leq i \leq k\right\}$ is a $G_{\omega}$-invariant partition of $\Delta$. Also, by Lemma 2.3 $G_{\omega}$ is transitive on $\mathcal{P}(\omega)$ and hence also on $\left\{\Delta \cap P_{i}(\omega) \mid 1 \leq i \leq k\right\}$. Thus the sets $\Delta \cap P_{i}(\omega)$ all have the same size; so $|\Delta|$ is divisible by $k$.

Lemma 2.5. For positive integers $n$ and $k$, if there exists a $k$-TOD of degree $n$, then $n \equiv 1(\bmod k)$, and in particular, $k$ is coprime to $n$. Moreover, if the $k-T O D$ is symmetric and $n$ is odd, then $n \equiv 1(\bmod 2 k)$.

Proof. Let $(M, G, \Omega, \mathcal{P})$ be a $k$-TOD of degree $n$, and let $\mathcal{P}=\left\{P_{1}, P_{2}, \ldots, P_{k}\right\}$. By Lemma 2.4, it follows that $k$ divides the length of each $G_{\omega}$-orbit in $\Omega \backslash\{\omega\}$, and hence $k$ divides $n-1$.

Now assume that $\mathcal{P}$ is symmetric. Then each $P_{i}$ is a symmetric relation on $\Omega$, and so there are $n(n-1) /(2 k)$ unordered pairs $\{\alpha, \beta\}$ such that $P_{i}$ contains $(\alpha, \beta)$ and $(\beta, \alpha)$. If $n$ is odd, then $2 k$ is coprime to $n$ since $k$ is coprime to $n$, and thus $(n-1) /(2 k)$ is an integer, that is, $n \equiv 1(\bmod 2 k)$. 


\section{Some Construction methods for TODs}

3.1. TODs and partitions of $\operatorname{Orbl}(M, \Omega)$. Suppose that $(M, G, \Omega, \mathcal{P})$ is a $k$ TOD with $M \triangleleft G$. Then, for each $G$-orbit $Q$ in $\operatorname{Orbl}(M, \Omega)$, the partition $\mathcal{P}$ of $\Omega^{(2)}$ determines a partition $\mathcal{B}(Q)=\{B(P) \mid P \in \mathcal{P}\}$ of $Q$, where $B(P)$ is the set of $M$-orbitals $\Delta \in Q$ such that $\Delta \subseteq P$. The next result explores the connection between the existence of a $k$-TOD and the existence of $k$-part partitions $\mathcal{B}(Q)$ for the $G$-orbits $Q$ in $\operatorname{Orbl}(M, \Omega)$. This result underlies our later constructions of cyclic TODs in Section 6.

Actions of a group $G$ on $\Omega$ and $\Omega^{\prime}$ are said to be permutationally equivalent if there is a bijection $f: \Omega \rightarrow \Omega^{\prime}$ such that, for all $g \in G$ and $\omega \in \Omega,\left(\omega^{g}\right) f=(\omega f)^{g}$. For a transitive subgroup $M \leq \operatorname{Sym}(\Omega)$ and a subset $X \subseteq \operatorname{Orbl}(M, \Omega)$, define $X^{*}=\left\{\Delta^{*} \mid \Delta \in X\right\}$. We say that $X$ is symmetric if $X=X^{*}$.

Construction 3.1. Let $M$ be a transitive permutation group on $\Omega$, and let $M \unlhd$ $G \leq \operatorname{Sym}(\Omega)$. Suppose that for each $G$-orbit $Q$ in $\operatorname{Orbl}(M, \Omega)$, there exists a $G$ invariant partition $\mathcal{B}(Q)$ of $Q$ with $k$ parts. Choose a particular $G$-orbit $Q$ and let $B \in \mathcal{B}(Q)$. Suppose that the actions induced by $G$ on the $\mathcal{B}(Q)$ are permutationally equivalent, that is, for any other $G$-orbit $Q^{\prime}$ in $\operatorname{Orbl}(M, \Omega)$, there exists a bijection $f_{Q^{\prime}}: \mathcal{B}(Q) \rightarrow \mathcal{B}\left(Q^{\prime}\right)$ such that, for all $C \in \mathcal{B}(Q)$ and all $g \in G,\left(C^{g}\right) f_{Q^{\prime}}=\left(C f_{Q^{\prime}}\right)^{g}$. Let $J=\left\{g_{1}, \ldots, g_{k}\right\}$ be a set of coset representatives for the setwise stabiliser $G_{B}$ in $G$, and note that $G_{B}=G_{(B) f_{Q^{\prime}}}$ for each $Q^{\prime}$. Define $P_{1}$ to be the union, over all $G$-orbits $Q^{\prime}$ in $\operatorname{Orbl}(M, \Omega)$, of all ordered pairs of points contained in $M$-orbitals in (B) $f_{Q^{\prime}}$; that is,

$$
P_{1}=\bigcup_{Q^{\prime}}\left(\bigcup_{\Delta \in(B) f_{Q^{\prime}}} \Delta\right)
$$

Let $P_{i}=P_{1}^{g_{i}}$ for $i=1, \ldots, k$, and let $\mathcal{P}=\left\{P_{1}, \ldots, P_{k}\right\}$.

That the 4-tuple $(M, G, \Omega, \mathcal{P})$ produced by this construction is a TOD is proved below.

Lemma 3.2. For $M, G, \mathcal{P}$ as in Construction 3.1, $(M, G, \Omega, \mathcal{P})$ is a $k$-TOD, and moreover it is symmetric if and only if

(a) for each symmetric orbit $R$, each part of the partition $\mathcal{B}(R)$ is symmetric, and

(b) for each nonsymmetric orbit $R$, and each $B \in \mathcal{B}(Q)$, we have $\left(B f_{R}\right)^{*}=$ (B) $f_{R^{*}}$.

Proof. Suppose that $(M, G, \Omega, \mathcal{P})$ is a tuple produced in Construction 3.1. Since $G_{B}=G_{(B) f_{Q^{\prime}}}$, it follows that $\mathcal{B}\left(Q^{\prime}\right)=\left\{\left(B f_{Q^{\prime}}\right)^{g_{i}} \mid i=1, \ldots, k\right\}$ for each $Q^{\prime}$, and therefore $\mathcal{P}$ is a partition of $\Omega^{(2)}$. Moreover,

$$
P_{i}=\bigcup_{Q^{\prime}}\left(\bigcup_{\Delta \in B f_{Q^{\prime}}} \Delta\right)^{g_{i}}=\bigcup_{Q^{\prime}}\left(\bigcup_{\Delta^{\prime} \in\left(B f_{Q^{\prime}}\right)^{g_{i}}} \Delta^{\prime}\right)=\bigcup_{Q^{\prime}}\left(\bigcup_{\Delta^{\prime} \in\left(B^{g_{i}}\right) f_{Q^{\prime}}} \Delta^{\prime}\right) .
$$

Let $g \in G$. Then a similar argument gives $P_{i}^{g}=\bigcup_{Q^{\prime}}\left(\bigcup_{\Delta \in\left(B^{g_{i} g}\right) f_{Q^{\prime}}} \Delta\right)=P_{j}$, where $B^{g_{i} g}=B^{g_{j}}$. Thus $\mathcal{P}$ is $G$-invariant, and $G^{\mathcal{P}}$ is permutationally equivalent to $G^{\mathcal{B}(Q)}$ and, in particular, is transitive. Thus $(M, G, \Omega, \mathcal{P})$ is a $k$-TOD.

Suppose that $(M, G, \Omega, \mathcal{P})$ is a symmetric $k$-TOD, and let $R$ be a symmetric orbit and $B \in \mathcal{B}(R)$. Let $\Delta \in B$, and suppose that $\Delta \subset P$ with $P \in \mathcal{P}$. Since $R$ is symmetric we have $\Delta^{*} \in R$, and since $(M, G, \Omega, \mathcal{P})$ is symmetric we have $\Delta^{*} \subseteq P$. 
By Construction $3.1 B$ consists of all orbitals $\Delta^{\prime}$ such that $\Delta^{\prime} \in R$ and $\Delta^{\prime} \subseteq P$. Therefore $\Delta^{*} \in B$. It follows that $B=B^{*}$ is symmetric. Let $R$ be a nonsymmetric orbit. Let $\Delta \in R$ lie in the block $B f_{R}$ of $\mathcal{B}(R)$. Then $\Delta^{*} \in\left(B f_{R}\right)^{*}$ and $\Delta^{*} \in R^{*}$. Suppose that $\Delta \in P, P \in \mathcal{P}$. Since $\mathcal{P}$ is symmetric, we have $\Delta^{*} \in P$. By the definition of $f_{R^{*}},(B) f_{R^{*}}$ is the set of orbitals $\Delta^{*} \in R^{*}$ that are contained in $P$. Hence $\Delta^{*} \in(B) f_{R^{*}}$, and so $\left(B f_{R}\right)^{*}=(B) f_{R^{*}}$.

Conversely, suppose that, for each symmetric orbit $R$, each part of the partition $\mathcal{B}(R)$ is symmetric and for each nonsymmetric orbit $R$ and $C \in \mathcal{B}(Q),(C) f_{R^{*}}=$ $\left(C f_{R}\right)^{*}$. We claim that $(M, G, \Omega, \mathcal{P})$ is symmetric. Since $G^{\mathcal{P}}$ is transitive, it is sufficient to prove that $P_{1}=P_{1}^{*}$. Let $\Delta \subseteq P_{1}$ lie in an orbit $R$. If $R$ is symmetric, then $B f_{R}$ is the set of orbitals $\Delta^{\prime} \in R$ such that $\Delta^{\prime} \in P_{1}$. Hence $\Delta \in B f_{R}$. Now $\Delta^{*} \in R$ since $R$ is symmetric, and $\Delta^{*} \in B f_{R}$ since the part $B f_{R}$ of $\mathcal{B}(R)$ is symmetric, and hence $\Delta^{*} \subseteq P_{1}$. Now let $R$ be not symmetric. Again $\Delta \in B f_{R}$; so $\Delta^{*} \in\left(B f_{R}\right)^{*}=(B) f_{R^{*}}$ and, by Construction 3.1. $\Delta^{*} \subseteq P_{1}$. Thus $P_{1}=P_{1}^{*}$; so $(M, G, \Omega, \mathcal{P})$ is symmetric.

Now we obtain a set of necessary and sufficient conditions for the existence of TODs based on the action on $\operatorname{Orbl}(M, \Omega)$.

Proposition 3.3. $\quad$ (i) Let $M$ be a transitive permutation group on $\Omega$, and let $M \unlhd G \leq \operatorname{Sym}(\Omega)$. Then there exists a partition $\mathcal{P}$ of $\Omega^{(2)}$ such that $(M, G, \Omega, \mathcal{P})$ is a $k$-TOD if and only if for each $G$-orbit $Q$ in $\operatorname{Orbl}(M, \Omega)$, there exists a $G$-invariant partition $\mathcal{B}(Q)$ of $Q$ with $k$ parts, and the actions of $G$ on $\mathcal{B}(Q)$, for all $G$-orbits $Q$ in $\operatorname{Orbl}(M, \Omega)$, are pairwise permutationally equivalent.

(ii) Moreover, there exists a symmetric $k$-TOD $(M, G, \Omega, \mathcal{P})$ if and only if, in addition, for each symmetric $G$-orbit $Q$ in $\operatorname{Orbl}(M, \Omega)$ (if any such exists), there exists a partition $\mathcal{B}(Q)$ as in part (i), each part of which is symmetric.

Proof. Suppose that there exists $\mathcal{P}=\left\{P_{1}, \ldots, P_{k}\right\}$ such that $(M, G, \Omega, \mathcal{P})$ is a $k$ TOD. Let $J=\left\{g_{1}, \ldots, g_{k}\right\} \subset G$ be such that $P_{1}^{g_{i}}=P_{i}$ for each $i$. Since $G^{\mathcal{P}}$ is transitive, each $G$-orbit $Q$ in $\operatorname{Orbl}(M, \Omega)$ contains (at least one) $M$-orbital $\Delta \subseteq P_{1}$. Let $B_{1}$ be the set of $M$-orbitals $\Delta$ such that $\Delta \in Q$ and $\Delta \subseteq P_{1}$. For each $i$, set $B_{i}=B_{1}^{g_{i}}$. Then $B_{i}$ is the set of $M$-orbitals $\Delta$ such that $\Delta \in Q$ and $\Delta \subseteq$ $P_{i}$. Thus $\mathcal{B}(Q)=\left\{B_{1}, \ldots, B_{k}\right\}$ is a $G$-invariant partition of $Q$ with $k$ parts, and $G^{\mathcal{B}(Q)}$ is permutationally isomorphic to $G^{\mathcal{P}}$. Conversely, if suitable $G$-invariant partitions $\mathcal{B}(Q)$ exist for each $Q$, then Construction 3.1 gives the required $k$-TOD by Lemma 3.2. Thus part (i) is proved.

Suppose further that $(M, G, \Omega, \mathcal{P})$ is symmetric, and suppose that $Q$ is a symmetric $G$-orbit in $\operatorname{Orbl}(M, \Omega)$. Then, since $P_{1}=P_{1}^{*}$, the set $B_{1}$ of $M$-orbitals $\Delta \in Q$ that are contained in $P_{1}$ satisfies $B_{1}^{*}=B_{1}$. By the definition of $\mathcal{B}(Q)$ above, each part of $\mathcal{B}(Q)$ is symmetric.

Conversely, suppose that for each symmetric orbit $Q$, there is a $\mathcal{B}(Q)$ with all parts symmetric. For any such $Q$, we choose a partition $\mathcal{B}(Q)$ with this extra property. Choose a particular $G$-orbit $Q$ in $\operatorname{Orbl}(M, \Omega)$, and for each $G$-orbit $R$ let $f_{R}: \mathcal{B}(Q) \rightarrow \mathcal{B}(R)$ be the bijection defining the permutational equivalence of the $G$-actions (taking $f_{Q}$ to be the identity map). Suppose that $R \neq R^{*}$. Then $R^{*}$ is also a $G$-orbit in $\operatorname{Orbl}(M, \Omega)$; so, in particular, $R \cap R^{*}=\emptyset$. If necessary we replace $\mathcal{B}\left(R^{*}\right)$ by $\mathcal{B}^{*}(R):=\left\{B^{*} \mid B \in \mathcal{B}(R)\right\}$, and we replace $f_{R^{*}}$ by $f_{R}^{*}$ : $\mathcal{B}^{*}(Q) \rightarrow \mathcal{B}^{*}(R)$ defined by $(B) f_{R}^{*}=\left(B f_{R}\right)^{*}$, for $B \in \mathcal{B}(Q)$. Let $(M, G, \Omega, \mathcal{P})$ be as 
in Construction 3.1 using these partitions $\mathcal{B}(R)$. Then by Lemma $3.2(G, M, \Omega, \mathcal{P})$ is a symmetric TOD.

3.2. Some TODs derived from a given one. Our first construction varies the partition $\mathcal{P}^{\prime}$ but involves the same subgroup $M$.

Lemma 3.4. Let $(M, G, \Omega, \mathcal{P})$ be a $k$-TOD.

(1) If $\mathcal{P}^{\prime}$ is a nontrivial $G$-invariant partition of $\operatorname{Orbl}(M, \Omega)$ refined by $\mathcal{P}$, then $k^{\prime}=\left|\mathcal{P}^{\prime}\right| \geq 2, k^{\prime}$ divides $k$, and $\left(M, G, \Omega, \mathcal{P}^{\prime}\right)$ is a $k^{\prime}-T O D$.

(2) If $H<G$ is such that $H^{\mathcal{P}}$ is semiregular and nontrivial with orbits of length $k^{\prime}$, and $H$ normalises $M$, then $k^{\prime} \mid k, k^{\prime} \geq 2$, and $\left(M,\langle M, H\rangle, \Omega, \mathcal{P}^{\prime}\right)$ is a $k^{\prime}$-TOD for some $H$-invariant partition $\mathcal{P}^{\prime}$ refined by $\mathcal{P}$.

Proof. (1). Since $G$ is transitive on $\mathcal{P}$ and $\mathcal{P}^{\prime}$ is refined by $\mathcal{P}, G$ is transitive on $\mathcal{P}^{\prime}$, and hence $\left(M, G, \Omega, \mathcal{P}^{\prime}\right)$ is a TOD of index $k^{\prime}$ dividing $k$.

(2). Choose a representative from each $H$-orbit in $\mathcal{P}$, and let $P_{1}^{\prime}$ be the union of these representatives. Set $\mathcal{P}^{\prime}=\left\{\left(P_{1}^{\prime}\right)^{h} \mid h \in H\right\}$. Since $H$ is semiregular on $\mathcal{P}$ with orbits of length $k^{\prime}$, it follows that $\mathcal{P}^{\prime}$ is an $H$-invariant partition of $\Omega^{(2)}$ with $k^{\prime}$ parts and refined by $\mathcal{P}$.

The next construction is the key both to a reduction to consideration of TODs $(M, G, \Omega, \mathcal{P})$ with $G$ primitive on $\Omega$, and also to the proof of Theorem 1.1] For a partition $\mathcal{P}$ of $\Omega^{(2)}$ and a subset $\Delta \subset \Omega$, by the restriction of $\mathcal{P}$ to $\Delta^{(2)}$ we mean the partition $\mathcal{Q}=\left\{P_{i} \cap(\Delta \times \Delta) \mid 1 \leq i \leq k\right\}$ of $\Delta^{(2)}$. Let $M \leq G \leq \operatorname{Sym}(\Omega)$ with $M$ transitive. For $\Delta \in \operatorname{Orbl}(M, \Omega)$, the paired orbital $\Delta^{*}=\{(\beta, \alpha) \mid(\alpha, \beta) \in \Delta\}$ also lies in $\operatorname{Orbl}(M, \Omega)\}$. If $G$ leaves $\operatorname{Orbl}(M, \Omega)$ invariant, then, for each $g \in G$, $\left(\Delta^{*}\right)^{g}=\left(\Delta^{g}\right)^{*}$. For a subgroup $N \leq \operatorname{Sym}(\Omega)$ and $\omega \in \Omega$, we denote by $\omega^{N}$ the $N$-orbit containing $\omega$.

Lemma 3.5. Let $(M, G, \Omega, \mathcal{P})$ be a $k$-TOD with $M$ normal in $G$, let $\omega \in \Omega$, and suppose that $N$ is a subgroup of $M$ with no fixed points in $\Omega$. Assume that $E \leq G_{\omega}$ is such that $E^{\mathcal{P}}$ is transitive and $E \leq \mathbf{N}_{G}(N)$. Set $F=N E$ and $\Delta=\omega^{N}$. Then the restriction $\mathcal{Q}$ of $\mathcal{P}$ to $\Delta^{(2)}$ is such that $\left(N^{\Delta}, F^{\Delta}, \Delta, \mathcal{Q}\right)$ is a $k$-TOD, and $E^{\mathcal{P}}$ is permutationally isomorphic to $F^{\mathcal{Q}}$. Further, if in addition $(M, G, \Omega, \mathcal{P})$ is symmetric, then also $\left(N^{\Delta}, F^{\Delta}, \Delta, \mathcal{Q}\right)$ is symmetric.

Proof. Let $\mathcal{P}=\left\{P_{1}, P_{2}, \ldots, P_{k}\right\}$; so $\mathcal{P}(\omega)=\left\{P_{1}(\omega), P_{2}(\omega), \ldots, P_{k}(\omega)\right\}$. Since $\mathcal{P}$ is refined by $\operatorname{Orbl}(M, \Omega)$, each of the $P_{i}(\omega)$ is $M_{\omega}$-invariant and hence also $N_{\omega^{-}}$ invariant. Thus each $Q_{i}(\omega):=\Delta \cap P_{i}(\omega)$ is $N_{\omega}$-invariant. Define $\mathcal{Q}(\omega)=\left\{Q_{i}(\omega) \mid\right.$ $1 \leq i \leq k\}$. Observe that $\bigcup_{i} Q_{i}(\omega)=\Delta \backslash\{\omega\}$, and if $i \neq j$, then $Q_{i}(\omega) \cap Q_{j}(\omega)=\emptyset$; so $\mathcal{Q}(\omega)$ is a partition of $\Delta \backslash\{\omega\}$. Also, $\mathcal{Q}(\omega)$ is invariant under $N_{\omega}$.

By assumption $E^{\mathcal{P}}$ is transitive, and since $E \leq G_{\omega}, E$ also acts transitively on $\mathcal{P}(\omega)$. Further, since $E$ normalises $N$ and fixes $\omega$, it follows that $E$ fixes $\Delta$ setwise. Thus, $E$ has an induced action on $Q(\omega)$ given by $Q_{i}(\omega)^{g}=\Delta \cap P_{i}(\omega)^{g}(g \in E$, $i \leq k)$, which is permutationally isomorphic to its actions on $\mathcal{P}(\omega)$ and $\mathcal{P}$. In particular, $E$ acts transitively on $\mathcal{Q}(\omega)$, and all the $Q_{i}(\omega)$ are nonempty. Note that $F_{\omega}=N_{\omega} E$ and the induced action on $\mathcal{Q}(\omega)$ satisfies $F_{\omega}^{\mathcal{Q}(\omega)}=\left(N_{\omega} E\right)^{\mathcal{Q}(\omega)}=E^{\mathcal{Q}(\omega)}$. By Lemma 2.3, the corresponding partition $\mathcal{Q}$ of $\Omega^{(2)}$ is $F$-invariant, and the $F$ action on $\mathcal{Q}$ is equivalent to the $F_{\omega}$-action on $\mathcal{Q}(\omega)$. Thus $F^{\mathcal{Q}}$ is permutationally isomorphic to $E^{\mathcal{P}}$, and $\left(N^{\Delta}, F^{\Delta}, \Delta, \mathcal{Q}\right)$ is a $k$-TOD. From the definition of $Q$ (preceding Lemma [2.3) it is clear that $Q_{i} \subseteq P_{i}$ for each $i$, and therefore $Q_{i}=P_{i} \cap(\Delta \times \Delta)$, for $1 \leq i \leq k$. 
Assume in addition that $(M, G, \Omega, \mathcal{P})$ is symmetric, that is, each $P_{i}$ is symmetric. Let $O \in \operatorname{Orbl}(M, \Omega)$, and let $\hat{O} \in \operatorname{Orbl}(N, \Delta)$ be such that $\hat{O}(\omega) \subseteq O(\omega)$. Then $\hat{O}^{*}(\omega) \subseteq O^{*}(\omega)$. Assume that $O(\omega) \subseteq P_{i}(\omega)$. Then, since $P_{i}$ is symmetric, $O(\omega) \cup$ $O^{*}(\omega) \subseteq P_{i}(\omega)$. By the definition of $Q_{i}(\omega), \hat{O}(\omega) \cup \hat{O}^{*}(\omega) \subseteq Q_{i}(\omega)$. It follows that $Q_{i}$ is symmetric, and so $\left(N^{\Delta}, F^{\Delta}, \Delta, \mathcal{Q}\right)$ is symmetric.

Our final construction is not elementary, since it relies on an application of the result of Fein, Kantor and Schacher [6] that a transitive permutation group contains a fixed-point-free element of prime-power order. This result relies on the finite simple group classification. Recall that we may always assume, for a TOD $(M, G, \Omega, \mathcal{P})$, that $M$ is normal in $G$ and hence that $G$ leaves $\operatorname{Orbl}(M, \Omega)$ invariant.

Theorem 3.6. If $(M, G, \Omega, \mathcal{P})$ is a $k$-TOD with $M$ normal in $G$, then there exists a $p$-TOD $(M, H, \Omega, \mathcal{Q})$ for some prime divisor $p$ of $k$ and some partition $\mathcal{Q}$ of $\Omega^{(2)}$ refined by $\mathcal{P}$, where $H=\langle M, \tau\rangle$ for some $\tau \in G_{\omega} \backslash M_{\omega}$ where $\omega \in \Omega$. In particular, $\tau$ fixes no element of $\operatorname{Orbl}(M, \Omega)$.

Proof. Let $(M, G, \Omega, \mathcal{P})$ be a $k$-TOD with $M$ normal in $G$. Let $\omega \in \Omega$. Since $M$ is transitive, $G=M G_{\omega}$; so $G^{\mathcal{P}}=G_{\omega}^{\mathcal{P}}$. Thus $G_{\omega}^{\mathcal{P}}$ is transitive, and it follows from [6] that, for some prime $p, G_{\omega}$ contains an element $\tau$ of $p$-power order such that $\tau^{\mathcal{P}}$ has no fixed points in $\mathcal{P}$. Label the parts of $\mathcal{P}$ as $P_{i j}$, so that the $i^{\text {th }}$-orbit of $\left\langle\tau^{\mathcal{P}}\right\rangle$ in $\mathcal{P}$ is $\left\{P_{i, j} \mid 1 \leq j \leq p^{a_{i}}\right\}, a_{i} \geq 1$, and $P_{i j}^{\tau}=P_{i, j+1}$ (reading the second subscript modulo $\left.p^{a_{i}}\right)$. For $l=1, \ldots, p$, define $Q_{l}=\bigcup_{i}\left(P_{i, l} \cup P_{i, l+p} \cup \cdots \cup P_{i, l-p+p^{a_{i}}}\right)$. Then $\mathcal{Q}=\left\{Q_{1}, \ldots, Q_{p}\right\}$ is permuted cyclically by $\tau$, and $\mathcal{Q}$ is a partition of $\Omega^{(2)}$ refined by $\mathcal{P}$. Thus $(M,\langle M, \tau\rangle, \Omega, \mathcal{Q})$ is a $p$-TOD. Since $\mathcal{Q}$ is refined by $\mathcal{P}$, it is also refined by $\operatorname{Orbl}(M, \Omega)$, and it follows that $\tau$ acts on $\operatorname{Orbl}(M, \Omega)$ with no fixed points.

This result has an immediate consequence:

Lemma 3.7. Let $(M, G, \Omega, \mathcal{P})$ be a $k$-TOD such that $G$ has a regular normal subgroup $N$ contained in $M$. Then $N$ is soluble.

Proof. By Lemma 2.2, $(N, G, \Omega, \mathcal{P})$ is a $k$-TOD, and then, by Theorem 3.6 there exists an element $\tau \in G \backslash N$ that fixes no element of $\operatorname{Orbl}(N, \Omega)$. Since $N$ is regular on $\Omega$, it follows that $\tau$ fixes no non-identity element of $N$. Thus the automorphism of $N$ induced by conjugation by $\tau$ is fixed-point-free, and hence $N$ is soluble, see [9, Thm. 1.48].

\section{TODS AND IMPRIMITIVE GROUP ACTIONS}

4.1. TODs on blocks of imprimitivity. We show that the induced configuration of a $k$-TOD $(M, G, \Omega, \mathcal{P})$ on a block $B$ of imprimitivity for $G$ on $\Omega$ is also a $k$-TOD. Let $(M, G, \Omega, \mathcal{P})$ be a $k$-TOD, and let $\mathcal{B}$ be a $G$-invariant partition of $\Omega$. Let $\mathcal{P}_{B}:=\left\{P_{1}^{B}, P_{2}^{B}, \ldots, P_{k}^{B}\right\}$, where $P_{i}^{B}:=P_{i} \cap(B \times B)$. Then each $P_{i}^{B}$ is a union of $M_{B^{-}}$orbitals on $B$, and $\mathcal{P}_{B}$ is a partition of $\mathcal{B}^{(2)}$. We denote the setwise stabilisers of $B$ in $M, G$ by $M_{B}$ and $G_{B}$, respectively.

Lemma 4.1. Let $(M, G, \Omega, \mathcal{P})$ be a $k$-TOD with $M$ normal in $G$. Then for a nontrivial block $B$ of imprimitivity for $G^{\Omega},\left(M_{B}^{B}, G_{B}^{B}, B, \mathcal{P}_{B}\right)$ is a $k$-TOD; further, $G^{\mathcal{P}}$ is permutationally isomorphic to $G_{B}^{\mathcal{P}_{B}}$.

Proof. The setwise stabiliser $M_{B}$ has no fixed points in $\Omega$ and is normalised by $G_{\omega}$. Also, since $M^{\mathcal{P}}=1$, we have that $G^{\mathcal{P}}=G_{\omega}^{\mathcal{P}}$ is transitive. The result now follows from Lemma 3.5 applied with $N=M_{B}, E=G_{\omega}$, and $\Delta=B$. 
By choosing $B$ to be a minimal block of imprimitivity, we may assume that the group $G_{B}^{B}$ is primitive. This suggests studying $k$-TODs $(M, G, \Omega, \mathcal{P})$ with $G$ primitive on $\Omega$.

Proposition 4.2. Let $(M, G, \Omega, \mathcal{P})$ be a $k$-TOD such that $G$ is primitive on $\Omega$. Then $G^{\Omega}$ is of O'Nan-Scott type HA, AS, SD, CD or PA (as defined in [22]).

Proof. This is an immediate consequence of Lemma 3.7.

Such TODs are investigated further in [10] and, in particular, a classification is obtained of cyclic $p^{a}$-TODs where $p$ is a prime. It is shown there, in particular, that there exist TODs corresponding to each of the five O'Nan-Scott types specified in the proposition.

4.2. Quotients of TODs. Let $(M, G, \Omega, \mathcal{P})$ be a TOD and let $\mathcal{B}$ be a $G$-invariant partition of $\Omega$. There is a natural map from $\Omega^{(2)}$ to $\mathcal{B} \times \mathcal{B}$ given by $\left(\omega, \omega^{\prime}\right) \rightarrow\left(B, B^{\prime}\right)$, where $\omega \in B \in \mathcal{B}$ and $\omega^{\prime} \in B^{\prime} \in \mathcal{B}$. This induces a map from subsets of $\Omega^{(2)}$ to subsets of $\mathcal{B} \times \mathcal{B}$. However, there are at least two reasons why, in general, a partition $\mathcal{P}$ of $\Omega^{(2)}$ is not mapped to a partition of $\mathcal{B}^{(2)}$. First there is the problem that distinct points $\omega, \omega^{\prime}$ may lie in the same block of $\mathcal{B}$. One might hope still to achieve a partition of $\mathcal{B}^{(2)}$ simply by ignoring such pairs. However, the second problem is that disjoint subsets of $\Omega^{(2)}$ may correspond to non-disjoint subsets of $\mathcal{B}^{(2)}$. This second problem makes it impossible, in general, to find a natural partition of $\mathcal{B}^{(2)}$ corresponding to a given partition of $\Omega^{(2)}$. In particular, there seems to be no natural construction of a TOD for the actions of $M$ and $G$ on $\mathcal{B}$ from a given TOD $(M, G, \Omega, \mathcal{P})$. This is demonstrated by the following simple example.

Example 4.3. Let $M=\langle(123456789)\rangle \cong \mathbb{Z}_{9}, G=\mathrm{D}_{18}$, and $\Omega=\{1,2, \ldots, 9\}$. Then $\operatorname{Orbl}(M, \Omega)=\left\{\Delta_{i}=(1, i)^{M} \mid 2 \leq i \leq 9\right\}$. Let $P_{1}=\Delta_{2} \cup \Delta_{3} \cup \Delta_{4} \cup \Delta_{5}$ and $P_{2}=\Delta_{6} \cup \Delta_{7} \cup \Delta_{8} \cup \Delta_{9}$. Then $\mathcal{P}=\left\{P_{1}, P_{2}\right\}$ is a partition of $\Omega^{(2)}$ and $(M, G, \Omega, \mathcal{P})$ is a 2-TOD. Now $B_{1}=\{1,4,7\}, B_{2}=\{2,5,8\}$ and $B_{3}=\{3,6,9\}$ form a $G$-invariant partition $\mathcal{B}$ of $\Omega$, but the images of both $P_{1}$ and $P_{2}$, under the map $\Omega^{(2)} \rightarrow \mathcal{B} \times \mathcal{B}$ defined above, are $\mathcal{B} \times \mathcal{B}$.

However, we show in the next section that, for a cyclic TOD $(M, G, \Omega, \mathcal{P})$ with $G$-invariant partition $\mathcal{B}$ of $\Omega$, it is possible to construct an induced quotient TOD on $\mathcal{B}$ of the same index. (This is Theorem 1.2 , stated in the introduction.)

\section{Cyclic TODs}

To prove Theorem 1.2 we first prove a very useful result about cyclic TODs. A similar result can be found in [11].

Proposition 5.1. Let $(M, G, \Omega, \mathcal{P})$ be a cyclic $k$-TOD and let $K$ be the kernel of the action of $G$ on $\mathcal{P}$. Then each element $\tau \in G \backslash K$ has exactly one fixed point in $\Omega$.

Proof. Let $\omega \in \Omega$. We have $G=K G_{\omega}$, and so $G=\langle K, \sigma\rangle$ for some $\sigma \in G_{\omega}$. Let $k=\prod_{i=1}^{r} p_{i}^{e_{i}}$ for distinct primes $p_{i}, e_{i} \geq 1$, and $r \geq 1$. Let $\tau \in G \backslash K$. Then there exists $i$ such that the order of $\tau$ modulo $K$ is divisible by $p_{i}$, and hence $\tau \notin\left\langle K, \sigma^{p_{i}^{e_{i}}}\right\rangle$. Let $\hat{\mathcal{P}}$ be the partition of $\Omega^{(2)}$ such that each part is the union of the parts of $\mathcal{P}$ contained in some orbit of $\left\langle\left(\sigma^{p_{i}^{e_{i}}}\right)^{\mathcal{P}}\right\rangle$. Then by Lemma 3.4 $(1),(M, G, \Omega, \hat{\mathcal{P}})$ is a cyclic $p_{i}^{e_{i}}$-TOD. Set $\hat{M}=\left\langle K, \sigma^{p_{i}^{e_{i}}}\right\rangle$. Then by Lemma $2.2(\hat{M}, G, \Omega, \hat{\mathcal{P}})$ is a cyclic 
$p_{i}^{e_{i}}$-TOD, and $\hat{M}$ is the kernel of the action of $G$ on $\hat{\mathcal{P}}$. There is a $p_{i}$-element $\sigma^{\prime}$ such that $G=\left\langle\hat{M}, \sigma^{\prime}\right\rangle=\langle\hat{M}, \sigma\rangle$ (taking $\sigma^{\prime}$ to be the " $p_{i}$-part" of $\sigma$ ).

Then $\tau=x\left(\sigma^{\prime}\right)^{l}$ for some $x \in \hat{M}$ and some integer $l$ not divisible by $p_{i}^{e_{i}}$. Since $x$ fixes $\hat{\mathcal{P}}$ pointwise, the $\langle\tau\rangle$-action on $\hat{\mathcal{P}}$ is equivalent to the action of $\left\langle\sigma^{l}\right\rangle$ on $\hat{\mathcal{P}}$. Thus $\langle\tau\rangle$ is nontrivial and half-transitive on $\hat{\mathcal{P}}$. In particular, $\tau$ fixes no element of $\hat{\mathcal{P}}$, and so $\tau$ fixes no $\left(\omega, \omega^{\prime}\right) \in \Omega^{(2)}$ for any distinct $\omega, \omega^{\prime} \in \Omega$. Hence $\tau$ fixes at most one point of $\Omega$.

If $\tau$ has $p_{i}$-power order, then, since $p_{i} \nmid|\Omega|$ by Lemma 2.5, $\tau$ fixes at least one point of $\Omega$, so that it fixes exactly one point of $\Omega$.

Suppose now that the order $o(\tau)=n_{1} n_{2}$ is such that $n_{1}$ is a $p_{i}$-power, $\operatorname{gcd}\left(n_{1}, n_{2}\right)$ $=1$, and $n_{2}>1$, and write $\tau=\tau_{1} \tau_{2}$ such that $o\left(\tau_{i}\right)=n_{i}$ and $\tau_{1} \tau_{2}=\tau_{2} \tau_{1}$. Since $|G: \hat{M}|=p_{i}^{e_{i}}$, it follows that $\tau_{2} \in \hat{M}$, and therefore $\tau_{1}$ is of $p_{i}$-power order and lies in $G \backslash \hat{M}$. By the argument of the previous paragraph, $\tau_{1}$ has exactly one fixed point in $\Omega$. Let $\Delta_{1}, \Delta_{2}, \ldots, \Delta_{t}$ be the $\langle\tau\rangle$-orbits in $\Omega$. Since $\left\langle\tau_{1}\right\rangle$ is a normal subgroup of $\langle\tau\rangle$, we have that $\left\langle\tau_{1}\right\rangle$ acts on each $\Delta_{j}$ half-transitively. Thus either $\left\langle\tau_{1}\right\rangle$ acts on $\Delta_{j}$ trivially, or $\left|\Delta_{j}\right|$ is divisible by $p_{i}$. Since $\tau_{1}$ has exactly one fixed point in $\Omega$, it follows that exactly one of the $\Delta_{j}$ has size 1 and all the others have size a multiple of $p_{i}$. Therefore, $\tau$ fixes exactly one point in $\Omega$.

Now we deduce a corollary for imprimitive cyclic TODs.

Lemma 5.2. Let $(M, G, \Omega, \mathcal{P})$ be a cyclic $k$-TOD. Let $K$ be the kernel of the $G$ action on $\mathcal{P}$, and let $\mathcal{B}$ be a nontrivial $G$-invariant partition of $\Omega$. Then each element of $G \backslash K$ fixes exactly one block of $\mathcal{B}$.

Proof. Here $G=\langle K, \sigma\rangle$ for some $\sigma \in G$ such that $\sigma^{k} \in K$ and $G / K \cong \mathbb{Z}_{k}$. Then, by Lemma 2.2. $(K, G, \Omega, \mathcal{P})$ is a cyclic $k$-TOD. Write $\mathcal{B}=\left\{B_{0}, B_{1}, \ldots, B_{t}\right\}$ for some $t \geq 2$. Let $\tau \in G \backslash K$. By Proposition [5.1 $\tau$ fixes a point of $\Omega$ and hence fixes setwise a block of $\mathcal{B}$, say $B_{0}$. Now $\tau=f \sigma^{r}$ for some integer $r$ and $f \in K$, and $\tau$ has order $k_{0}$ modulo $K$ for some $k_{0} \mid k$ with $k_{0}>1$. Since $K$ acts trivially on $\mathcal{P}$, $\left\langle\tau^{\mathcal{P}}\right\rangle=\left\langle\left(\sigma^{r}\right)^{\mathcal{P}}\right\rangle$ has $k / k_{0}$ orbits of length $k_{0}$ in $\mathcal{P}$. Let $\mathcal{P}=\left\{P_{1}, P_{2}, \ldots, P_{k}\right\}$, and observe that, for any $i \neq 0$,

$$
\begin{aligned}
& \left(P_{1} \cap\left(B_{0} \times B_{i}\right)\right) \cup \cdots \cup\left(P_{k} \cap\left(B_{0} \times B_{i}\right)\right)=B_{0} \times B_{i}, \text { and } \\
& \left(P_{j} \cap\left(B_{0} \times B_{i}\right)\right) \cap\left(P_{j^{\prime}} \cap\left(B_{0} \times B_{i}\right)\right)=\emptyset \quad \text { if } j \neq j^{\prime},
\end{aligned}
$$

where $B_{0} \times B_{i}=\left\{\left(\omega, \omega^{\prime}\right) \mid \omega \in B_{0}, \omega^{\prime} \in B_{i}\right\}$. Suppose that $\tau$ fixes $B_{i}$ setwise for some $i \in\{1,2, \ldots, t\}$. Then $\left(B_{0} \times B_{i}\right)^{\tau}=B_{0} \times B_{i}$, and for any $j \in\{1,2, \ldots, k\}$, there exists $j^{\prime} \in\{1,2, \ldots, k\}$ such that

$$
\left(P_{j} \cap\left(B_{0} \times B_{i}\right)\right)^{\tau}=P_{j^{\prime}} \cap\left(B_{0} \times B_{i}\right) .
$$

If $P_{j} \cap\left(B_{0} \times B_{i}\right) \neq \emptyset$, then for each of the $k_{0}$ parts $P_{j^{\prime}}$ in the $\left\langle\tau^{\mathcal{P}}\right\rangle$-orbit containing $P_{j}$, we have $\left|P_{j} \cap\left(B_{0} \times B_{i}\right)\right|=\left|P_{j^{\prime}} \cap\left(B_{0} \times B_{i}\right)\right|$. Since this is true for all $\left\langle\tau^{\mathcal{P}}\right\rangle$-orbits, it follows that $k_{0}$ divides $\left|B_{0} \times B_{i}\right|$. However, by Lemma $4.1\left(K_{B_{0}}, G_{B_{0}}^{B_{0}}, B_{0}, \mathcal{P}_{B_{0}}\right)$ is a $k$-TOD, and hence by Lemma $2.5\left|B_{0}\right| \equiv 1(\bmod k)$. Therefore, $\left|B_{0} \times B_{i}\right|=$ $\left|B_{0}\right|^{2} \equiv 1(\bmod k)$, and so $\left|B_{0} \times B_{i}\right| \equiv 1\left(\bmod k_{0}\right)$, which is a contradiction since $k_{0}>1$. Therefore, $B_{0}$ is the unique fixed block of $\tau$.

We are now ready to prove Theorem 1.2

Proof of Theorem 1.2. Let $K$ be the kernel of $G$ acting on $\mathcal{P}$. Then $G / K \cong \mathbb{Z}_{k}$, and $G=\langle K, \sigma\rangle$ for some $\sigma \in G$ such that $\sigma$ normalises $K$ and $\sigma^{k} \in K$. Since $K$ 
is transitive on $\Omega$, we have that $G=K G_{\omega}$, where $\omega \in \Omega$. Hence $\sigma=f \sigma^{\prime}$ where $f \in K$ and $\sigma^{\prime} \in G_{\omega}$. Since $f$ fixes every element of $\mathcal{P},\left\langle\sigma^{\prime}\right\rangle$ induces a transitive action on $\mathcal{P}$. Thus, without loss of generality, we may assume that $\sigma \in G_{\omega}$. Let $\mathcal{B}=\left\{B_{0}, B_{1}, \ldots, B_{t}\right\}$ be a $G$-invariant partition of $\Omega$ such that $\omega \in B_{0}$. Then in particular $B_{0}^{\sigma}=B_{0}$, and hence $\sigma$ normalises $K_{B_{0}}$. Let $\mathcal{D}$ be the set of $K_{B_{0}}$-orbits in $\Omega \backslash B_{0}$. Then $\mathcal{D}$ is $\langle\sigma\rangle$-invariant.

Let $\mathcal{P}=\left\{P_{1}, P_{2}, \ldots, P_{k}\right\}$ be such that $P_{i}^{\sigma}=P_{i+1}$ for each $i<k$ and $P_{k}^{\sigma}=P_{1}$, and let $\mathcal{P}\left(B_{0}\right)=\left\{P_{1}\left(B_{0}\right), P_{2}\left(B_{0}\right), \ldots, P_{k}\left(B_{0}\right)\right\}$, where

$$
P_{i}\left(B_{0}\right)=\left\{\alpha \in \Omega \backslash B_{0} \mid(\beta, \alpha) \in P_{i} \text {, for some } \beta \in B_{0}\right\} .
$$

Then

$$
P_{i}\left(B_{0}\right)^{\sigma}=\left\{\alpha^{\sigma} \in \Omega \backslash B_{0} \mid\left(\beta^{\sigma}, \alpha^{\sigma}\right) \in P_{i}^{\sigma}, \text { for some } \beta^{\sigma} \in B_{0}\right\}=P_{i+1}\left(B_{0}\right),
$$

reading the subscripts modulo $k$. Since $K_{B_{0}}$ acts trivially on $\mathcal{P}$, it follows that $K_{B_{0}}$ fixes each $P_{i}\left(B_{0}\right)$ setwise, and hence each $P_{i}(B)$ is a union of some subset of $\mathcal{D}$.

Suppose that $\Delta \in \mathcal{D}$ is contained in $P_{1}\left(B_{0}\right)$, and suppose that $B \in \mathcal{B} \backslash\left\{B_{0}\right\}$ is such that $B \cap \Delta$ contains a point $\alpha$. Suppose further that $1 \leq i \leq k$, and $B \cap \Delta^{\sigma^{i}}$ also contains a point, say $\beta$. Then $\beta=\delta^{\sigma^{i}}$ for some $\delta \in \Delta$, and since $K_{B_{0}}$ is transitive on $\Delta, \delta=\alpha^{g}$ for some $g \in K_{B_{0}}$. Thus $\beta=\alpha^{g \sigma^{i}} \in B \cap B^{g \sigma^{i}}$, and hence $g \sigma^{i}$ fixes $B$. However, $g \sigma^{i} \in G_{B_{0}}$ and $B \neq B_{0}$. It follows from Lemma 5.2 that $g \sigma^{i} \in K$. Hence $\sigma^{i} \in K$, and so $i=k$. Thus the $k$ sets $\Delta, \Delta^{\sigma}, \ldots, \Delta^{\sigma^{k-1}} \in \mathcal{D}$ meet disjoint subsets of $\mathcal{B} \backslash\left\{B_{0}\right\}$. Moreover, $\Delta^{\sigma^{i}} \subseteq\left(P_{1}\left(B_{0}\right)\right)^{\sigma^{i}}=P_{i+1}\left(B_{0}\right)$ for each $i<k$.

For a $K_{B_{0}}$-orbit $\Delta$, let $\mathcal{B}(\Delta)$ denote the subset of blocks $B$ of $\mathcal{B} \backslash\left\{B_{0}\right\}$ such that $B \cap \Delta \neq \emptyset$. Suppose that $\mathcal{B}(\Delta) \cap \mathcal{B}\left(\Delta^{\prime}\right)$ (where $\Delta, \Delta^{\prime} \in \mathcal{D}$ ) contains a block $B$, and let $B^{\prime}$ be an arbitrary block in $\mathcal{B}(\Delta)$. Then, since $B \cap \Delta \neq \emptyset, B^{\prime} \cap \Delta \neq \emptyset$, and $\Delta$ is a $K_{B_{0}}$-orbit, some element $x \in K_{B_{0}}$ maps a point of $B \cap \Delta$ to a point of $B^{\prime} \cap \Delta$, and hence $B^{x}=B^{\prime}$. Since $\Delta^{\prime}$ is a $K_{B_{0}}$-orbit, we have $\left(B \cap \Delta^{\prime}\right)^{x}=B^{\prime} \cap \Delta^{\prime}$, and therefore $B^{\prime} \in \mathcal{B}\left(\Delta^{\prime}\right)$. Thus $\mathcal{B}(\Delta) \subseteq \mathcal{B}\left(\Delta^{\prime}\right)$, and a similar argument proves that $\mathcal{B}\left(\Delta^{\prime}\right) \subseteq \mathcal{B}(\Delta)$. Thus, for $\Delta, \Delta^{\prime} \in \mathcal{D}, \mathcal{B}(\Delta)$ and $\mathcal{B}\left(\Delta^{\prime}\right)$ are either equal or disjoint. It may happen that $\mathcal{B}(\Delta)=\mathcal{B}\left(\Delta^{\prime}\right)$ for distinct $K_{B_{0}}$-orbits $\Delta, \Delta^{\prime}$, but we have just proved that, in this case, $\Delta$ and $\Delta^{\prime}$ lie in different $\langle\sigma\rangle$-orbits. Thus $\langle\sigma\rangle$ permutes the set $\{\mathcal{B}(\Delta) \mid \Delta \in \mathcal{D}\}$ with all orbits of length $k$. Suppose that $\langle\sigma\rangle$ has $m$ orbits in this set, and suppose, without loss of generality, that $\mathcal{B}\left(\Delta_{1}\right), \ldots, \mathcal{B}\left(\Delta_{m}\right)$ are representatives of these $m$ orbits. Define $Q_{1}\left(B_{0}\right):=\mathcal{B}\left(\Delta_{1}\right) \cup \cdots \cup \mathcal{B}\left(\Delta_{m}\right)$ and, for $2 \leq i \leq k$, set $Q_{i}\left(B_{0}\right):=\left(Q_{1}\left(B_{0}\right)\right)^{\sigma^{i-1}}$, and set $\mathcal{Q}\left(B_{0}\right):=\left\{Q_{i}\left(B_{0}\right) \mid 1 \leq i \leq k\right\}$. Then $\langle\sigma\rangle$ is transitive on $\mathcal{Q}\left(B_{0}\right)$, and it follows from Lemma 2.3 that $\left(M^{\mathcal{B}}, G^{\mathcal{B}}, \mathcal{B}, \mathcal{Q}\right)$ is a cyclic $k$-TOD, where $\mathcal{Q}=\left\{Q_{1}, \ldots, Q_{k}\right\}$ with $Q_{i}=\left\{\left(B_{0}, B\right)^{g} \mid B \in Q_{i}\left(B_{0}\right), g \in\right.$ $G\}$ (as defined before Lemma 2.3).

\section{EXPLICIT CONSTRUCTION FOR CYCLIC TODS}

For a cyclic $k$-TOD $(M, G, \Omega, \mathcal{P})$, we may assume by Lemma 2.2 that $M$ is normal in $G$ and therefore that there exists $\sigma \in G \backslash M$ such that $\sigma$ normalises $M$ and $\langle\sigma\rangle$ acts transitively on $\mathcal{P}$. The following is a consequence of Proposition 3.3 and gives a criterion for a transitive permutation group $M$ to give rise to a cyclic TOD. 
Lemma 6.1. Let $M$ be a transitive permutation group on $\Omega$, and let

$$
\sigma \in \mathbf{N}_{\operatorname{Sym}(\Omega)}(M) \text { and } G=\langle M, \sigma\rangle<\operatorname{Sym}(\Omega) .
$$

Then there exists a partition $\mathcal{P}$ of $\Omega^{(2)}$ such that

(i) $(M, G, \Omega, \mathcal{P})$ is a $k$-TOD if and only if $k$ divides the size of each $\langle\sigma\rangle$-orbit on $\operatorname{Orbl}(M, \Omega)$;

(ii) $(M, G, \Omega, \mathcal{P})$ is a symmetric $k$-TOD if and only if $k$ divides the size of each $\langle\sigma\rangle$-orbit on $\operatorname{Orbl}(M, \Omega)$, and for each $\Delta \in \operatorname{Orbl}(M, \Omega)$ and each $\tau \in$ $\langle\sigma\rangle \backslash\left\langle\sigma^{k}\right\rangle, \Delta^{\tau} \neq \Delta^{*}$, where $\Delta^{*}$ is the paired orbital of $\Delta$.

Proof. Part (i) follows immediately from Proposition 3.3. For part (ii), the extra condition is that, for each symmetric $\langle\sigma\rangle$-orbit $Q$ in $\operatorname{Orbl}(M, \Omega)$, there exists a symmetric $k$-part partition $\mathcal{B}(Q)$ with appropriate $\langle\sigma\rangle$-action. Now $Q$ is a symmetric $\langle\sigma\rangle$-orbit if and only if $Q=Q^{*}$, that is, $\Delta^{*} \in Q$ whenever $\Delta \in Q$. We require that each part $B \in \mathcal{B}(Q)$ should be symmetric, that is, $\Delta^{*} \in B$ whenever $\Delta \in B$. Since $\mathcal{B}(Q)=\left\{B^{\sigma^{i}} \mid 0 \leq i<k\right\}$, this condition implies that, for each $\Delta \in \operatorname{Orbl}(M, \Omega)$, $\Delta^{\sigma^{i}} \neq \Delta^{*}$ for $i=1, \ldots, k-1$ (either because $\Delta$ and $\Delta^{*}$ lie in different $\langle\sigma\rangle$-orbits, or because they lie in the same block $B$ of $\mathcal{B}(Q)$, where $\left.\Delta, \Delta^{*} \in Q\right)$. Conversely, the condition $\Delta^{\sigma^{i}} \neq \Delta^{*}$ for all $\Delta$ and for $i=1, \ldots, k-1$ enables us to define a $G$-invariant partition with all parts symmetric, for each symmetric $G$-orbit $Q$. (Take $B=\left\{\Delta^{\sigma^{k i}} \mid i \geq 0\right\}$.)

If an element $\sigma \in \mathbf{N}_{\mathrm{Sym}(\Omega)}(M)$ has $p$-power order with $p$ prime, and $\sigma$ fixes no element of $\operatorname{Orbl}(M, \Omega)$, then $p$ divides the size of every $\langle\sigma\rangle$-orbit on $\operatorname{Orbl}(M, \Omega)$. Suppose that $\tau$ is an automorphism of $M$ that normalises a point stabilizer in $M$. Then $\tau$ is induced by some element $\tilde{\tau} \in \operatorname{Sym}(\Omega)$ such that $\tilde{\tau} \in \mathbf{N}_{\operatorname{Sym}(\Omega)}(M)$. Thus $\tilde{\tau}$ acts on $\operatorname{Orbl}(M, \Omega)$. By Lemma 6.1 , we have a criterion for a transitive permutation group to have a TOD of prime index in terms of certain special automorphisms of the group.

Corollary 6.2. A transitive group $M$ acting on $\Omega$ has a TOD of prime index $p$ if and only if $M$ has an automorphism $\tau$ of p-power order such that $\tau$ normalises some point stabiliser in $M$, and $\tilde{\tau}$ fixes no element of $\operatorname{Orbl}(M, \Omega)$, where $\tilde{\tau}$ is as above.

To conclude this section, we construct explicit examples of cyclic TODs for all values of $n$ and $k$ occurring in Theorem 1.1

Construction 6.3. Let $n=r_{1}^{d_{1}} \ldots r_{m}^{d_{m}}$, where the $r_{i}$ are distinct primes, $d_{i} \geq 1$ and $m \geq 1$. Let $M=\mathbb{Z}_{r_{1}}^{d_{1}} \times \cdots \times \mathbb{Z}_{r_{m}}^{d_{m}}$. Then $\operatorname{Aut}(M)=\prod_{i=1}^{m} \operatorname{GL}\left(d_{i}, r_{i}\right) \geq$ $\prod_{i=1}^{m} \mathrm{GL}\left(1, r_{i}^{d_{i}}\right)$.

(a) Suppose that $r_{i}^{d_{i}} \equiv 1(\bmod k)$ for each $i$. Choose $\sigma_{i} \in \operatorname{GL}\left(1, r_{i}^{d_{i}}\right)$ such that $\sigma_{i}$ is of order $k$. Let $\sigma=\sigma_{1} \ldots \sigma_{m} \in \operatorname{Aut}(M)$. Take $P_{1}(1)$ to consist of one representative of each of the $\langle\sigma\rangle$-orbits in $M \backslash\{1\}$, and set

$$
\begin{aligned}
& P_{1}=\left\{(x, y) \mid x, y \in M, x y^{-1} \in P_{1}(1)\right\}, \text { and } \\
& \mathcal{P}=\left\{P_{1}^{\sigma^{i}} \mid 0 \leq i<k\right\} .
\end{aligned}
$$

(b) Suppose that $r_{i}^{d_{i}} \equiv 1(\bmod 2 k)$ whenever $r_{i}$ is odd, and $r_{i}^{d_{i}} \equiv 1(\bmod k)$ if $r_{i}=2$. For each $i$, if $r_{i}$ is odd, then let $\sigma_{i} \in \mathrm{GL}\left(1, r_{i}^{d_{i}}\right)$ have order $2 k$, and if $r_{i}=2$, 
let $\sigma_{i} \in \mathrm{GL}\left(1, r_{i}^{d_{i}}\right)$ have order $k$. Let $\sigma=\sigma_{1} \sigma_{2} \ldots \sigma_{r} \in \operatorname{Aut}(M)$. Take $P_{1}(1)$ to consist of one representative of each of the $\langle\sigma\rangle$-orbits in $M \backslash\{1\}$, and set

$$
\begin{aligned}
& P_{1}=\left\{(x, y) \mid x, y \in M, x y^{-1} \in P_{1}(1)\right\}, \text { and } \\
& \mathcal{P}=\left\{P_{1}^{\sigma^{i}} \mid 0 \leq i<k\right\} .
\end{aligned}
$$

The next lemma shows that these constructions produce cyclic $k$-TODs.

Lemma 6.4. (i) For $M, \sigma, \mathcal{P}, n, k$ as in Construction 6.3 (a), $(M,\langle M, \sigma\rangle, M$, $\mathcal{P})$ is a cyclic $k-T O D$ of degree $n$; if in addition $k$ is odd, then $(M,\langle M, \sigma\rangle$, $M, \mathcal{P})$ is a symmetric cyclic $k$-TOD of degree $n$.

(ii) For $M, \sigma, \mathcal{P}, n, k$ as in Construction 6.3(b), $(M,\langle M, \sigma\rangle, M, \mathcal{P})$ is a symmetric cyclic $k-T O D$ of degree $n$.

Proof. The cyclic group GL $\left(1, r_{i}^{d_{i}}\right)$ is regular on $\mathbb{Z}_{r_{i}}^{d_{i}} \backslash\{1\}$, and hence $\prod_{i=1}^{m} \operatorname{GL}\left(1, r_{i}^{d_{i}}\right)$ is semiregular on $M \backslash\{1\}$. Thus in both constructions (given in Construction 6.3) $\langle\sigma\rangle$ acts semiregularly on $M \backslash\{1\}$, and so $\langle\sigma\rangle$ acts on $\operatorname{Orbl}(M, M)$ with all orbits of length $k$ in part (i) and length $2 k$ or $k$ in part (ii). It follows from Lemma 6.1 that in both cases, $(M,\langle M, \sigma\rangle, M, \mathcal{P})$ is a cyclic $k$-TOD.

Now suppose that $M, \sigma, \mathcal{P}, n, k$ are as in Construction 6.3 (a) with $k$ odd, or as in Construction 6.3(b). We show that the condition of Lemma6.1(ii) holds. Suppose to the contrary that $\Delta^{\sigma^{i}}=\Delta^{*}$, where $\Delta \in \operatorname{Orbl}(M, M)$ and $\sigma^{i} \notin\left\langle\sigma^{k}\right\rangle$. Then in particular $k \nmid i$. Now $\Delta(1)=\{x\}$ for some $x \in M \backslash\{1\}$, and $\Delta^{*}(1)=\left\{x^{-1}\right\}$. Since $\sigma^{i}$ fixes $1, x^{\sigma^{i}}=x^{-1}$, and so $x^{\sigma^{2 i}}=x$. Now all $\langle\sigma\rangle$-orbits in $M \backslash\{1\}$ have length $2 k$ or $k$. Since $x^{\sigma^{2 i}}=x$, we have that $k \mid 2 i$. Since $k \nmid i, k$ must be even. Thus the proof of part (i) is complete. Continuing with the proof of part (ii), by Lemma 2.5 $n$ is odd. It then follows from the definition of $\sigma$ that all $\langle\sigma\rangle$-orbits in $M \backslash\{1\}$ have length $2 k$. Hence $2 k \mid 2 i$ and $k \mid i$, which is a contradiction. So $\Delta^{\sigma^{i}} \neq \Delta^{*}$, satisfying Lemma 6.1 (ii).

\section{Degrees And indices}

In this section, we prove a relation between index $k$ and degree $n$ for a cyclic $k$-TOD of degree $n$, and complete the proof of Theorem 1.1. First, we show that if $(M, G, \Omega, \mathcal{P})$ is a cyclic TOD, then some Sylow subgroups induce cyclic TODs. For a prime $r$, by $r^{a} \| n$ we mean that $r^{a}$ is the highest power of $r$ dividing $n$.

Lemma 7.1. Let $(M, G, \Omega, \mathcal{P})$ be a cyclic $p^{e}-T O D$ of degree $n$ with $M$ normal in $G$, where $p$ is a prime and $G=\langle M, \sigma\rangle$ for some element $\sigma$ of p-power order. Let $r$ be a prime such that $r^{d} \| n$ with $d>0$, and let $R$ be a Sylow $r$-subgroup of $M$. Then there exist an element $\sigma_{0} \in G$, an orbit $\Sigma$ of $R$ in $\Omega$, and a partition $\mathcal{Q}$ of $\Sigma^{(2)}$ such that $\left(R,\left\langle R, \sigma_{0}\right\rangle, \Sigma, \mathcal{Q}\right)$ is a cyclic $p^{e}-T O D$ of degree $r^{d}$. If in addition $(M, G, \Omega, \mathcal{P})$ is symmetric, then $\left(R,\left\langle R, \sigma_{0}\right\rangle, \Sigma, \mathcal{Q}\right)$ is also symmetric.

Proof. There exists $l \geq d$ such that $r^{l}=|R|$, so that $r^{l-d}$ is the order of a Sylow $r$-subgroup of $M_{\omega}$, where $\omega \in \Omega$. Let $\tau$ be an arbitrary element of $G \backslash\left\langle M, \sigma^{p}\right\rangle$. Then $\left\langle M, \sigma^{p}, \tau\right\rangle=G$. By Lemma $5.1 \tau$ fixes a unique point in $\Omega$, and we denote the point by $\omega_{\tau}$. Thus $\tau \in G_{\omega_{\tau}}$, and it follows that $\tau$ normalises the point-stabilizer $M_{\omega_{\tau}}$.

Let $\rho \in G \backslash\left\langle M, \sigma^{p}\right\rangle$, and let $S$ be a Sylow $r$-subgroup of $M_{\omega_{\rho}}$. Then $|S|=r^{l-d}$, and $S^{\rho}$ is also a Sylow $r$-subgroup of $M_{\omega_{\rho}}$. By Sylow's theorem, $S^{\rho}=S^{g}$ for some $g \in M_{\omega_{\rho}}$. Thus $S^{\rho^{\prime}}=S$, where $\rho^{\prime}:=\rho g^{-1} \in G \backslash\left\langle M, \sigma^{p}\right\rangle$. Since both $\rho$ and $g$ fix $\omega_{\rho}$, 
we have that $\rho^{\prime}$ fixes $\omega_{\rho}$. Hence by Lemma 5.1, $\omega_{\rho^{\prime}}=\omega_{\rho}$. Thus $S$ is an $r$-subgroup of $M$ for which there exists an element $\rho^{\prime} \in G \backslash\left\langle M, \sigma^{p}\right\rangle$ such that $S^{\rho^{\prime}}=S$ and $\left|S_{\omega_{\rho^{\prime}}}\right|=r^{l-d}$.

Assume now that $X$ is maximal by inclusion among $r$-subgroups of $M$ such that there exists $\tau \in G \backslash\left\langle M, \sigma^{p}\right\rangle$ satisfying

$$
X^{\tau}=X \quad \text { and } \quad\left|X_{\omega_{\tau}}\right|=r^{l-d} .
$$

Let $N=\mathbf{N}_{M}(X)$, and let $Y$ be a Sylow $r$-subgroup of $N$. Note that $N$ has no fixed points in $\Omega$, for if $S$ is a Sylow $r$-subgroup of $M$ containing $X$, then $\mathbf{N}_{S}(X)$ properly contains $X$, and hence has no fixed points in $\Omega$. Now $\tau$ normalises $N$, $X \unlhd Y$, and $Y^{\tau}$ is a Sylow $r$-subgroup of $N$. Thus $Y^{\tau}=Y^{x}$ for some $x \in N$, and so $Y^{\hat{\tau}}=Y$, where $\hat{\tau}:=\tau x^{-1}$. Let $\Delta=\omega_{\tau}^{N}$, the orbit of $N$ containing $\omega_{\tau}$. Since $\tau$ fixes $\omega_{\tau}$ and normalises $N$, we have that $\tau$ fixes $\Delta$ setwise. Thus, in particular, $\Delta^{\hat{\tau}}=\Delta^{\tau x^{-1}}=\Delta$. By Lemma 3.5. $\left(N,\langle N, \tau\rangle, \Delta, \mathcal{P}^{\prime}\right)$ is a cyclic $p^{e_{-}}$ TOD for some partition $\mathcal{P}^{\prime}$ of $\Delta^{(2)}$. Thus by Lemma [5.1, $\hat{\tau}$ fixes a point of $\Delta$, and so $\omega_{\hat{\tau}} \in \Delta$. Since $N$ is transitive on $\Delta$, we have $\omega_{\tau}^{y}=\omega_{\hat{\tau}}$ for some $y \in N$, and thus $X_{\omega_{\tau}}^{y} \leq N_{\omega_{\tau}}^{y}=N_{\omega_{\hat{\tau}}}$. So $X_{\omega_{\tau}}^{y} \leq X^{y} \cap N_{\omega_{\hat{\tau}}}=X \cap N_{\omega_{\hat{\tau}}}=X_{\omega_{\hat{\tau}}}$. Hence $r^{l-d}=\left|X_{\omega_{\tau}}\right|=\left|X_{\omega_{\tau}}^{y}\right| \leq\left|X_{\omega_{\hat{\tau}}}\right| \leq\left|Y_{\omega_{\hat{\tau}}}\right|$. However, since $r^{l-d}||\left|M_{\omega_{\hat{\tau}}}\right|$, we have that $\left|Y_{\omega_{\hat{\tau}}}\right|=r^{l-d}$. Therefore, $Y$ is an $r$-subgroup of $M$ such that $Y^{\hat{\tau}}=Y$ and $\left|Y_{\omega_{\hat{\tau}}}\right|=r^{l-d}$. By the maximality of $X$, we have $Y=X$. Thus $X$ is a Sylow $r$-subgroup of $\mathbf{N}_{M}(X)$, and hence $X$ is a Sylow $r$-subgroup of $M$. In particular, $|X|=r^{l}$ and $X$ has no fixed points in $\Omega$.

By Sylow's theorem, $R=X^{g}$ for some $g \in M$. Let $\tau_{0}=\tau^{g}$. Then by Lemma 3.5 applied to $R$ and $K=\left\langle R, \tau_{0}\right\rangle$, there exists a $p^{e}$-TOD $\left(R,\left\langle R, \tau_{0}\right\rangle, \Sigma, \mathcal{Q}\right)$, where $\Sigma=\omega_{\tau_{0}}^{R}$ and $|\Sigma|=\left|R: R_{\omega_{\tau_{0}}}\right|=r^{d}$. If in addition $(M,\langle M, \sigma\rangle, \Omega, \mathcal{P})$ is symmetric, then by Lemma [3.5, $\left(R,\left\langle R, \tau_{0}\right\rangle, \Sigma, \mathcal{Q}\right)$ is symmetric.

We now give a relation between the degrees and indices of cyclic $k$-TODs in the case where $k$ is a prime-power.

Lemma 7.2. Let $p$ be a prime, and let $n=r_{1}^{d_{1}} r_{2}^{d_{2}} \ldots r_{m}^{d_{m}}$ where the $r_{i}$ are distinct primes.

(i) If there exists a $p^{e}$-TOD of degree $n$, then $r_{i}^{d_{i}} \equiv 1\left(\bmod p^{e}\right)$, for all $r_{i}$.

(ii) If there exists a symmetric $p^{e}-$ TOD of degree $n$, then $r_{i}^{d_{i}} \equiv 1\left(\bmod 2 p^{e}\right)$ for all odd $r_{i}$, and $r_{i}^{d_{i}} \equiv 1\left(\bmod p^{e}\right)$ if $r_{i}=2$.

Proof. Let $(M, G, \Omega, \mathcal{P})$ be a cyclic $p^{e}$-TOD of degree $n$, where $G=\langle M, \sigma\rangle$ for some element $\sigma \in G$ of $p$-power order. Let $r$ be a prime such that $r^{d} \| n$ with $d>0$. Let $R$ be a Sylow $r$-subgroup of $M$. By Lemma 7.1 there exists a $p^{e}$-TOD $(R,\langle R, \tau\rangle, \Sigma, \mathcal{Q})$ of degree $r^{d}$. Thus, by Lemma 2.5], $r^{d} \equiv 1\left(\bmod p^{e}\right)$. If in addition $(M,\langle M, \sigma\rangle, \Omega, \mathcal{P})$ is symmetric, then by Lemma 7.1, $(R,\langle R, \tau\rangle, \Sigma, \mathcal{Q})$ is symmetric. Thus by Lemma 2.5, either $r^{d} \equiv 1\left(\bmod 2 p^{e}\right)$, or $r=2$ and $2^{d} \equiv 1\left(\bmod p^{e}\right)$.

We are now ready to prove Theorem 1.1

Proof of Theorem 1.1. If $k$ is a prime-power, then Theorem 1.1 follows from Lemmas 6.4 and 7.2 Thus we may assume that $k$ is not a prime-power. Let $(M, G, \Omega, \mathcal{P})$ be a cyclic $k$-TOD of degree $n$. Write $k=p_{1}^{e_{1}} p_{2}^{e_{2}} \ldots p_{l}^{e_{l}}$, where $p_{i}$ are distinct primes, $e_{i} \geq 1$, and $l \geq 2$. Let $\sigma \in G \backslash M$ be such that $\langle\sigma\rangle$ is transitive on $\mathcal{P}$. Recall that we may take $\sigma \in G_{\omega}$. Let $k_{i}=k / p_{i}^{e_{i}}$, and let $\sigma_{i}=\sigma^{k_{i}}$. Then $\left\langle\sigma_{i}\right\rangle$ acts half-transitively 
on $\mathcal{P}$, and each $\left\langle\sigma_{i}\right\rangle$-orbit on $\mathcal{P}$ has size $p_{i}^{e_{i}}$. Hence $\left\langle\sigma_{i}\right\rangle$ acts on the set of $M$-orbitals such that each orbit has size divisible by $p_{i}^{e_{i}}$. By Lemma 6.1 there exists a cyclic $p_{i}^{e_{i}}$-TOD $\left(M,\left\langle M, \sigma_{i}\right\rangle, \Omega, \mathcal{P}_{i}\right)$ for some partition $\mathcal{P}_{i}$ of $\Omega^{(2)}$. By Lemma 7.2 , if $r$ is a prime and $r^{d}|| \Omega \mid$, then $r^{d} \equiv 1\left(\bmod p_{i}^{e_{i}}\right)$. It then follows that $r^{d} \equiv 1(\bmod k)$.

Assume further that $(M, G, \Omega, \mathcal{P})$ is symmetric. By Lemma 3.5 (taking $N=M$ and $\left.E=\langle\sigma\rangle \leq G_{\omega}\right),\left(M,\left\langle M, \sigma_{i}\right\rangle, \Omega, \mathcal{P}_{i}\right)$ is symmetric. By Lemma 7.2 if $r$ is odd and $r^{d}|||\Omega|$, then $r^{d} \equiv 1\left(\bmod 2 p_{i}^{e_{i}}\right)$. It then follows that $r^{d} \equiv 1(\bmod 2 k)$ if $r$ is odd.

The converse assertion of Theorem 1.1 follows from Lemma 6.4.

\section{REFERENCES}

[1] B. Alspach, J. Morris and V. Vilfred, Self-complementary circulant graphs, Ars Combinatoria 53 (1999), 187-191. MR 2001b:05184

[2] P. Cameron, Finite permutation groups and finite simple groups, Bull. London Math. Soc 13 (1981), 1-22. MR 83m:20008

[3] N. J. Calkin, P. Erdös and C. A. Tovey, New Ramsey bounds from cyclic graphs of prime order, Siam J. Discrete Math. 10 (1997), 381-387. MR 98e:05078

[4] V. Chvátal, P. Erdös and Z. Hedrlín, Ramsey's theorem and self-complementary graphs, Disc. Math. 3(1972), 301-304. MR 47:1674

[5] C. R. J. Clapham, A class of self-complementary graphs and lower bounds of some Ramsey numbers, J. Graph Theory 3 (1979), 287-289. MR 81d:05054

[6] B. Fein, W. M. Kantor and M. Schacher, Relative Brauer groups II. J. Reine Angew. Math. 328 (1981), 39-57. MR 83a:12018

[7] M. Fried, R. Guralnick and J. Saxl, Schur covers and Carlitz's conjecture, Israel J. Math. 82 (1993), 157-225. MR 94j:12007

[8] D. Fronček, A. Rosa and J. Širan, The existence of self-complementary circulant graphs, European J. Combin. 17 (1996), 625-628. MR 97d:05235

[9] D. Gorenstein, Finite Simple Groups, 1982, Plenum Press, New York. MR 84j:20002

[10] R. M. Guralnick, C. H. Li, C. E. Praeger and J. Saxl, On orbital partitions and exceptionality of primitive permutation groups, preprint.

[11] R. M. Guralnick, P. Müller and J. Saxl, The rational function analogue of a question of Schur and exceptionality of permutation representations, Mem. Amer. Math. Soc. (to appear)

[12] F. Harary, R. W. Robinson and N. C. Wormald, Isomorphic factorisations I: Complete graphs. Trans. Amer. Math. Soc. 242 (1978), 243-260. MR 58:27646a

[13] F. Harary and R. W. Robinson, Isomorphic factorisations X: unsolved problems, J. Graph Theory 9 (1985), 67-86. MR 87a:05111

[14] R. Jajcay and C. H. Li, Constructions of self-complementary circulants with no multiplicative isomorphisms, European J. Combin. 22 (2001), 1093-1100. MR 2002g:05098

[15] C. H. Li, On self-complementary vertex-transitive graphs, Comm. Algebra 25 (1997), 39033908. MR 98k:05119

[16] C. H. Li, T. K. Lim and C. E. Praeger, Homogeneous factorisations of complete graphs with edge-transitive factors, in preparation.

[17] C. H. Li and C. E. Praeger, Self-complementary vertex-transitive graphs need not be Cayley graphs, Bull. London Math. Soc. 33 (2001), 653-661. MR 2002h:05085

[18] V. Liskovets and R. Pöschel, Non-Cayley-isomorphic self-complementary circulant graphs, J. Graph Theory 34 (2000), 128-141. MR 2001e:05056

[19] R. Mathon, On selfcomplementary strongly regular graphs, Disc. Math. 69 (1988), 263-281. MR 89d:05150

[20] M. Muzychuk, On Sylow subgraphs of vertex-transitive self-complementary graphs, Bull. London Math. Soc. 31 (1999), 531-533. MR 2001i:05093

[21] W. Peisert, All self-complementary symmetric graphs, J. Algebra 240 (2001), 209-229. MR 2002e:05074

[22] C. E. Praeger, Finite quasiprimitive graphs, Surveys in Combinatorics 1997 (London), 6585, London Math. Soc. Lecture Notes series 241, (Cambridge Univ. Press, Cambridge, 1997). MR 99b:05076 
[23] S. B. Rao, On regular and strongly regular selfcomplementary graphs, Disc. Math. 54 (1985), 73-82. MR 86g:05089

[24] H. Sachs, Über selbstkomplementäre Graphen, Publ. Math. Debrecen 9 (1962), 270-288. MR 27:1934

[25] D. A. Suprunenko, Selfcomplementary graphs, Cybernetics 21 (1985), 559-567. MR 87g:05197

[26] M. Suzuki, Group Theory I, (Springer, New York 1982). MR 82k:20001c

[27] B. Zelinka, Self-complementary vertex-transitive undirected graphs, Math. Slovaca 29 (1979), 91-95. MR 81h:05116

[28] H. Zhang, Self-complementary symmetric graphs, J. Graph Theory 16 (1992), 1-5. MR 92k:05061

Department of Mathematics and Statistics, The University of Western Australia, Crawley, WA 6009, Australia

E-mail address: li@maths.uwa.edu.au

Department of Mathematics and Statistics, The University of Western Australia, Crawley, WA 6009, Australia

E-mail address: praeger@maths.uwa.edu.au 